\title{
DETERMINACIÓN DE LAS FASES DE COLAPSO EN LAS DIFERENTES ETAPAS DE LA EVOLUCIÓN HISTÓRICO-AMBIENTAL DE CERRO DE PASCO (1901- 2008)
}

\section{DETERMINING COLLAPSE PHASES IN THE DIFFERENT STAGES OF THE ENVIRONMENTAL HISTORY EVOLUTION OF CERRO DE PASCO (1901-2008)}

\author{
Armando Aramayo Bazzetti ${ }^{1}$ y Edgar Sánchez Infantas ${ }^{2}$
}

\begin{abstract}
Resumen
Se analizó a la ciudad de Cerro de Pasco desde la llegada de la Cerro de Pasco Investment Company (después Cerro de Pasco Copper Corporation) en 1901 hasta el año 2008. Mediante los ciclos adaptativos, cuyo instrumento operativo es el análisis de los distintos capitales (que son los stocks que un sistema tiene, tales como el físico, natural, social, humano, político y el financiero o económico), se ha determinado un ciclo completo y un segundo casi por completarse. El primero de los ciclos se inicia con el colapso de la minería local y la caída del precio de la plata, lo que proporciona el impulso para que entre la gran minería a la región. La identificación de las fases colapso $(\Omega)$ se realiza mediante el análisis de estos seis capitales que se han tomado en consideración. Cada una de las variables funciona a su vez como un microciclo y cuando sucede que varios de ellos entran en esta fase colapso de manera conjunta o simultáneamente, entonces el sistema completo estaría entrando en la fase colapso, lo que significa que todo el sistema estaría entrando en un período de descomposición antes de reorganizarse e iniciar un nuevo período o ciclo. El planteamiento realizado en el presente estudio puede servir para identificar los distintos ciclos de sistemas socio-ecológicos de una manera más concluyente.
\end{abstract}

Palabras clave: Cerro de Pasco, minería, capitales, ciclo adaptativo.

\begin{abstract}
The present study analyzes the city of Cerro de Pasco since the arrival of Cerro de Pasco Investment Company (afterwards named Cerro de Pasco Copper Corporation) in 1901 up to the year 2008. Using the adaptive cycles, whose operational instrument is the analysis of the different capitals (which are the stocks a systems has, such as physical, natural, social, human, political and financial or economic), one complete cycle has been determined and an almost complete second one. The first one of these starts with the collapse of the local mining and the falling prices of silver, which gives the impulse for the entrance of big mining into the region. The identification of the collapse phases $(\Omega)$ is done by the analysis of six different capitals which have been taken into account. Each one of those is at the same time a microcycle and when several of them have entered that collapse phase, it means that the complete system would be entering the collapse phase, which means that the whole system is entering a period of decomposition before reorganizing and starting a new period or cycle. The proposal in the present study can be used to identify the different cycles in a more conclusive manner.
\end{abstract}

Key words: Cerro de Pasco, mining, capitals, adaptive cycle.

\section{Introducción.}

La evolución de Cerro de Pasco se ha caracterizado por tener épocas de bonanza y épocas difíciles. Ésta ha sido una ciudad minera desde sus inicios. En 1901 llega a la cuidad la Cerro de Pasco Investment Company y según Thorp \& Bertram (1988), la entrada de la compañía norteamericana fue el factor que dominaría, no sólo el desarrollo de la minería, sino el de toda la economía del Perú central. Siendo la entrada de la Cerro de Pasco Investment Company un evento tan importante tanto para el desarrollo de la minería como de la economía del Perú y de la región, en consecuencia debía serlo para el desarrollo de la ciudad de Cerro de Pasco, razón por la cual este momento se tomó como el inicio de la primera etapa del estudio. Por otro lado, la llegada de esta empresa (posteriormente llamada Cerro de Pasco Copper Corporation - CPCC) al Perú determinó una reestructuración de la minería nacional, la cual estaba, hasta ese entonces, liderada por unos cuantos grupos de familias que mantenían todavía fuertes intereses en el sector agrario (Quispe, 2006).

Ciclo adaptativo es un término extraído de la ecología, y utilizado para el análisis de los ecosistemas, cuyo modelo fue inicialmente propuesto 
por Holling (Abel, 1998; Weeks et al., 2004). El ciclo adaptativo plantea la existencia de cuatro fases (Resilience Alliance, 2008). Allí se representa lo que normalmente ocurre en un sistema ecológico, desde que se inicia, como consecuencia de algún colapso, para posteriormente reorganizarse, crecer hasta llegar a un clímax para finalmente desorganizarse nuevamente y recomenzar un nuevo ciclo.

Ahora la idea de los ciclos adaptativos está siendo utilizada cada vez más para temas no ecológicos. Según Abel et al. (2006) la teoría del ciclo adaptativo puede ser utilizada para examinar la dinámica y la resiliencia de los sistemas ecológico-sociales. En un breve análisis a la potencial utilidad que se le da a este instrumento, Weeks et al. (2004), tomaron cada una de las cuatro fases y las adaptaron a los sistemas sociales de la siguiente manera:

r: organización de un nuevo sistema de los restos del anterior

$\mathrm{K}$ : mantenimiento y proliferación del nuevo sistema

$\Omega$ : revolución

$\alpha$ : cambio de régimen/un nuevo paradigma

En su estudio, Weeks et al. (2004) explican como la teoría del ciclo adaptativo puede ser aplicada a hechos sociales tales como la revolución mejicana. Según estos, durante los años previos a Porfirio Díaz, Méjico se mantuvo por mucho tiempo en la fase $r$. Díaz llevó a la nación a una fase $\mathrm{K}$ y el colapso ocurrió con la insistencia de Francisco Madero de realizar nuevas elecciones, que llevó al país a la revolución. El sistema se desorganizó y recién se empezó a entrar en una fase alfa en 1916, con elecciones libres y con la creación de un nuevo sistema en el país, en donde se le dio tierras y voto a los campesinos. Aunque algunas cosas del sistema anterior se mantuvieron, las novedades en el nuevo sistema fueron la tenencia de la tierra por los campesinos y las elecciones democráticas.

Por otro lado, Bush (2004) desarrolla su estudio utilizando el análisis de capitales para determinar la evolución histórica y de cómo la percepción ambiental y el desarrollo influencian el uso, la gestión y la evolución de los recursos acuáticos en el contexto de un país con un pobre sistema de información. Indica que para determinar cómo los recursos determinan la sostenibilidad es necesario evaluar los capitales del que las sociedades dependen. Estos son el capital humano, capital social, capital físico, capital natural, y el capital financiero o económico. Baumann \& Sinha (2001) determinan que los capitales no deben ser cinco sino seis, siendo el sexto el capital político. Ellos explican que los cinco capitales son la base dentro del marco del modo de vida sostenible y que son sustituibles en parte por las personas. Pero estudios de campo en la India llevaron a los autores a incluir al capital político como el sexto de los capitales considerados pilar, ya que la lucha por el poder influye en el bienestar de la gente y por ello la dimensión política es un factor clave en el análisis de lo que está ocurriendo y de lo que pueda ocurrir con los grupos humanos estudiados. Los conflictos socioambientales son altamente políticos, y este factor es un instrumento para lograr el acceso a los recursos, razón por la que se ha decidido incluir este sexto elemento en el esquema de análisis para poder determinar el momento en el que se encuentra cada uno de los capitales señalado durante la fase $\Omega$.

Cuando un sistema entra en la fase $\Omega$ (omega) o de colapso, ésta es una etapa en la que la pérdida de capital es tal, que para su reorganización de los capitales se requiere une reorganización interna o una inyección de capitales de otro nivel, lo que permitiría que el sistema pueda reorganizarse o transformarse en algo diferente pero deseable (Abel et al., 2006). Mediante el colapso se comienza un nuevo ciclo, que puede contener un mismo atractor, o en caso que el sistema no se reorganice de manera semejante al estado anterior, habría un nuevo centro de gravedad, un elemento sobre el que gira todo el sistema.

La evolución histórica del conflicto socioambiental existente en la ciudad de Cerro de Pasco tiene distintas fases. Existe un estudio previo, de Dammert \& Carhuaricra (2003), en donde señalan que las etapas entre la llegada de la Cerro de Pasco Investment Company y el año 1999 son tres. Estos autores las subdividen de la siguiente manera:

- 1era etapa - desde la entrada de la empresa Cerro de Pasco Corporation hasta los años 50.

- 2da etapa - desde el inicio del tajo abierto en los años 50 hasta los años 90.

- 3era etapa - se inicia cuando la empresa CENTROMIN intentó reubicar a la ciudad de manera infructuosa, siendo impedido por las autoridades locales y la población en conjunto.

En el presente estudio se van a considerar los momentos que cambiaron la dinámica de la ciudad, a partir de la llegada de la CPCC hasta el año 2008 mediante el uso del ciclo adaptativo, para demostrar que la subdivisión anteriormente propuesta, en donde el infructuoso intento de CENTROMIN de reubicar la ciudad fue señalado como el momento gravitante entre una etapa y la siguiente, no es la correcta, y que mediante la utilización de los ciclos adaptativos se puede probar que es el tajo abierto lo que mantiene la dinámica del sistema y no la empresa que lo maneja o explota. 


\section{Materiales y métodos.}

Etapa 1 - Recopilación de información.

Se recopiló información sobre variables que puedan ser utilizadas como indicadores de los distintos capitales que conforman el ciclo adaptativo, tales como el capital natural, capital humano, capital social, capital físico, capital económico y capital político. Los capitales antes mencionados se analizaron mediante las variables presentadas en cada uno de los momentos históricos, según su relevancia o a la existencia de datos o información en cada uno de los casos.

Se realizó la recopilación de información histórica para determinar fechas y eventos clave que influenciaron los eventos en la ciudad de Cerro de Pasco y que permitieron hacer el análisis de la dinámica socio-ecológica de la ciudad (Seixas, 2002). Se realizó la búsqueda en lugares como:

- Municipio de Cerro de Pasco - mapa y publicaciones locales

- INC Pasco - mediante conversación con las personas cercanas a las fuentes de información, se pudo adquirir libros y se determinaron lugares en donde se pudo acceder a otras fuentes de información, tales como puestos de periódico cerca al Mercado Modelo de Chaupimarca en donde se consiguió información de publicaciones locales.

- Biblioteca Nacional

- Puesto de venta de libros antiguos en Lima

- Revistas locales en puestos de periódicos de Cerro de Pasco

- INEI - Perú Compendio Estadístico 2007; Departamento de Pasco: Compendio estadístico 2009; Perú: Migraciones internas 1993 - 2007.

- ONG LABOR - fotocopias de textos de su biblioteca, información y estadísticas en físico y en internet (en su página web).

- Otras mediante Internet

Etapa 2 - Determinación de los momentos críticos y validación mediante análisis de capitales

Mediante revisión histórica se realizó una revisión de los momentos críticos que tuvo la ciudad de Cerro de Pasco, para lo cual se utilizaron textos históricos y estudios anteriores que sirvieron de guía inicialmente. Posteriormente se buscó validar o invalidar este primer análisis mediante la utilización de los seis capitales mencionados anteriormente, analizando la fluctuación de las variables que componen los capitales durante ese cambio de fases.

Se determinaron los momentos $\Omega$ dentro de cada ciclo mediante el análisis de los 6 capitales, que están descritos en la Tabla 1. Cada uno de ellos tiene una serie de variables, y se utilizaron las que suministraron información que permitió determinar fluctuaciones en las mismas.
Tabla 1. Descripción de los 6 capitales.

Capital humano: $\begin{aligned} & \text { Aptitudes, } \\ & \text { capacidades laborales y buena salud. }\end{aligned}$

Capital social: Redes y conexiones, participación en grupos formales (adhesión a reglas, normas y sanciones acordadas de forma mutua y comúnmente aceptadas, relaciones de confianza, reciprocidad e intercambios).

Capital natural: $\quad$ Acceso y calidad de recursos naturales (agua, tierra, árboles, animales).

Capital físico: Infraestructuras básicas y bienes de producción (red vial y medios de transporte, comunicaciones, suministro de agua y energía, edificios, equipos y herramientas)

Capital financiero Disponibilidad de dinero (ahorros, : créditos, pensiones, remesas).

Capital político: Acceso al poder político, influencia política

Fuente: elaboración propia a partir de Suárez (2008) y Baumann \& Sinha (2001).

Análisis de los distintos capitales

El análisis del capital natural se calculó mediante la variación del mineral existente en la zona en estudio. Se determinó el incremento o disminución de los stocks - en términos de minerales explotados bajo determinada tecnología -, o la variación en la cantidad de mineral explotado. Esto depende de la cantidad de los stocks existentes, de la tecnología disponible o de alguna serie de factores que tengan control sobre las cantidades explotadas.

El análisis del capital físico se realizó a través de la evolución de la infraestructura física de la ciudad de Cerro de Pasco, siendo la base física el ámbito de residencia de la población, que además es un factor determinante en los capitales social y humano. Se tomó a las urbanizaciones y a las viviendas de la urbe y a su evolución en el tiempo dentro del casco urbano como las variables principales a considerar como capital físico.

El capital financiero se va a evaluar desde el aspecto económico de la población, de la empresa minera, o del gobierno local. La decisión de determinar cuál de estos tres va a ser la principal variable a tomar en cuenta, ha dependido de cuál de ellas ha sido la que primara.

Por otro lado, el capital humano, tal como se describe en Suárez (2008), tiene variables tales como aptitudes, conocimientos, capacidades laborales y buena salud, pero no todas son medibles en cada caso que se vaya a analizar. En las primeras etapas este capital se mide mejor según Reis (2001), quien explica que la mejor manera de analizar esta variable en el siglo XIX es mediante las capacidades laborales 
o aptitudes para el trabajo, mientras que la alfabetización o el nivel escolar logrado no es un factor que prime para lograr mejoras en los logros personales. Para el análisis de la última etapa del estudio, las variables escolaridad y la variable salud pueden expresar más claramente el futuro desarrollo personal de la población, y ambos tienen la ventaja de poder ser evaluados con cifras. La salud se ha determinado mediante la contaminación por plomo, ya que está demostrado que este metal pesado es un elemento que dentro de la sangre de los niños impide las sinapsis neuronales, lo que reduciría la capacidad cognitiva de estos y su posterior desarrollo cuando lleguen a ser adultos (Virginia Department of Health, 2004; Nation \& Gleaves, 2001). También se ha utilizado el indicador NBI (necesidades básicas insatisfechas) como un indicador del capital humano. Este utiliza la información de los censos demográficos y de vivienda para caracterizar la pobreza. Mediante el NBI se constata si los hogares satisfacen o no algunas de sus necesidades principales, y a partir de ello se construye un mapa de pobreza (Feres \& Mancero, 2001).

El capital social se analizó utilizando información que sirva para determinar el grado de cohesión que exista en la ciudad. Debido a la condición de ser ciudad minera, una variable muy importante en el análisis son los trabajadores mineros y las organizaciones que de ello se deriven debido a que estos trabajadores son una clase por sí mismos. La existencia de contratas mineras debilita la cohesión debido a que no existe la sindicalización, pero además influye en el capital político, porque un sindicato fuerte en una ciudad eminentemente minera es también una fuerza política. Otra variable que también influye sobre el capital social por un lado pero también sobre el capital humano es la migración. La migración debilita la cohesión social, pero si además éste incluye a los jóvenes en edad escolar también se debilita la formación de capital humano.

El capital político está representado por la empresa minera como tal, ya que sin importar el nombre de la empresa minera o empresas que estén en la ciudad, la industria funciona como enclave, lo que lleva a que ejerzan gran influencia sobre la variación de los capitales, tanto en lo social como en lo político. La población organizada en sindicatos, o trabajando de manera unida con una finalidad común, o una ONG o institución que funja de guía, pueden ser otros ejemplos de capital político, y su influencia en el tiempo puede cambiar, logrando que este capital suba o baje (en términos de lo que beneficie a la ciudad). Por otro lado, la aparición de determinadas normas también puede ejercer influencia sobre la dirección de las variables, ya que una norma tiene en sí misma influencia sobre el capital político.

Se determinó los cambios de los distintos capitales de manera cualitativa, en base a la comparación con la etapa anterior, y de esta manera evaluar el incremento o la disminución de las mismas.

\section{Resultados y discusión.}

Las etapas halladas en el presente estudio - desde la llegada de la Cerro de Pasco Investment Company (luego Cerro de Pasco Copper Corporation) hasta el año 2008 - forman un ciclo completo y uno casi por completar. Se tomaron estos momentos como los límites entre una etapa y la siguiente debido a que históricamente representan cambios significativos en la dinámica existente entre la ciudad y la minería. Las etapas encontradas se presentan a continuación:

\section{Etapa 0 La minería antes de la llegada de Cerro} de Pasco Mining

Etapa 1 Desde la llegada de la Cerro de Pasco Mining hasta el inicio de operaciones del tajo abierto

Etapa 2 Desde el inicio del tajo abierto hasta la actualidad (2008)

Se ha considerado una Etapa 0 en el análisis debido a que es importante determinar que el inicio de la Etapa 1 sea un momento de cambio en la dinámica de la ciudad de Cerro de Pasco y no una fecha arbitraria con poco significado en la evolución de la misma.

Los resultados, en términos de ciclos adaptativos se presenta en la Figura 1, en donde se señalan los momentos críticos y los puntos de quiebre de cada ciclo en el sistema, resultados que serán explicados en los siguientes párrafos.

Fines de la Etapa 0

Los eventos de fines de la Etapa 0 fueron subdivididos en los diferentes tipos de capital para entender el fenómeno de manera más completa. En términos de capital natural $(\mathrm{Kn})$ tenemos, por un lado, que el ciclo de la plata estaba en decadencia debido a los precios internacionales (Samamé, 1980; Kent, 1995), pero al mismo tiempo el precio del cobre estaba permitiendo que parte de los productores mineros derivaran a esta segunda alternativa (Barrios, 2008; Guadalupe et al., 2006). Un grave problema se presenta con la escasez de insumos como la madera que era necesaria para construir el ferrocarril que permitiría el transporte de todo el mineral a menor costo. Además había un número fijo de arrieros, lo que hacía que la cantidad de cobre que podría transportarse hacia las fundiciones y después hacia la costa para la exportación era limitada, poniendo de esta manera otra barrera más a la producción a gran escala del cobre (Contreras, 2004). Tomando en cuenta estos elementos se puede considerar que el Kn estaba en decadencia.

Según Contreras (2004), hubieron varios problemas: primero, los costos de transporte eran muy elevados, ya que los arrieros que llevaban el mineral 
cobraban montos muy elevados, y como no había suficiente oferta de estos, al incrementarse la presión por producir más, la falta de transporte se convirtió en un gran obstáculo para el posterior desarrollo de la minería local y de la producción. En segundo lugar, los mineros tenían mucha dificultad para encontrar operarios para sus operaciones y por último, tenían dificultad para conseguir insumos claves, como es el caso de la madera. quita fuerza frente al gigante que se venía, que era la Cerro de Pasco Copper. Habría que incluir en esto la lucha que había de los arrieros contra cualquier evento que pudiera alterar la ventaja del que gozaban con el monopolio del transporte (Contreras, 2004). La poca cohesión entre los actores locales y el poco peso de los mineros para hacer presión al gobierno determinan que el Ks sea bajo, así como el Kp (capital político).

El capital financiero (Kf) también está en

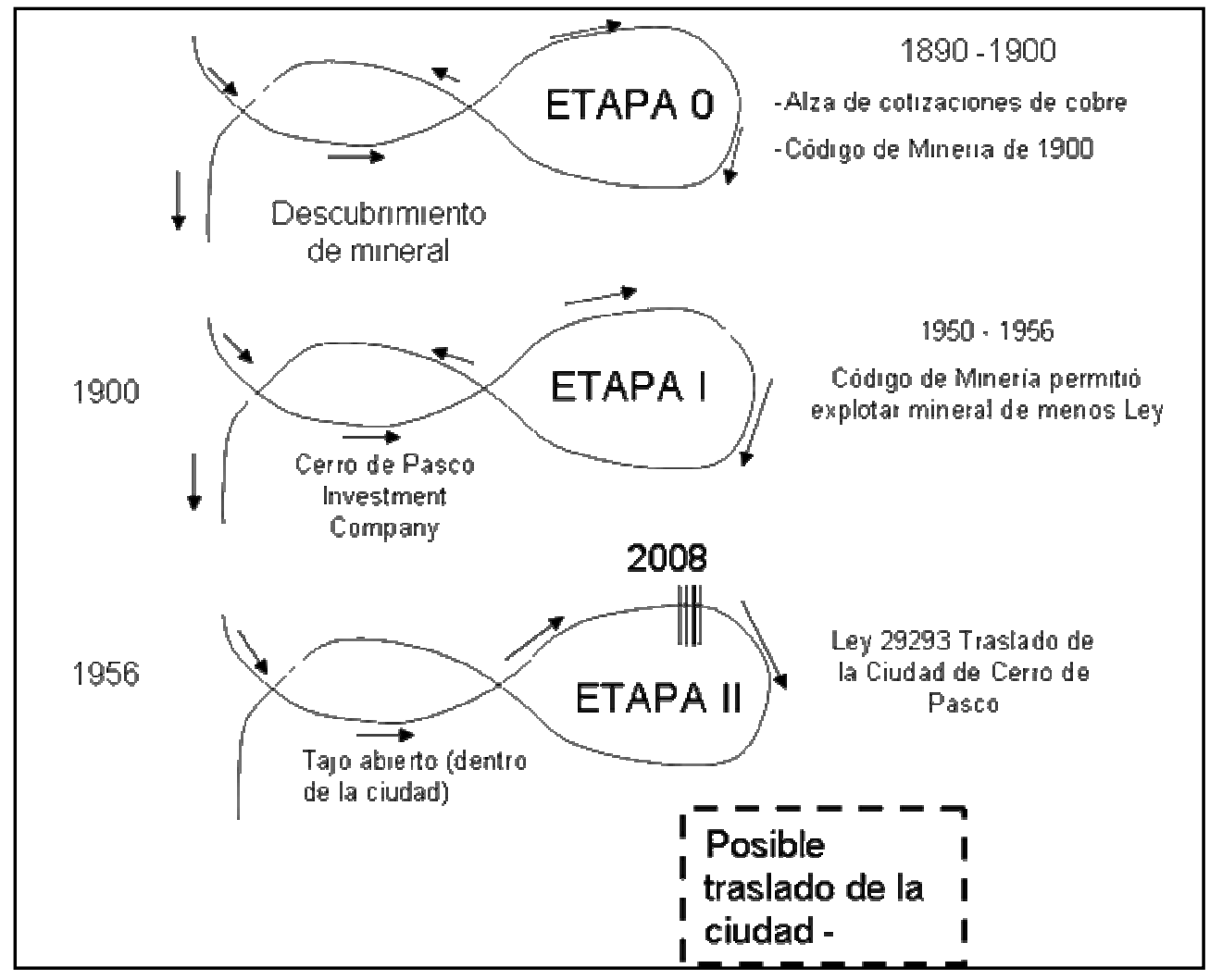

Figura 1. Ciclos adaptativos durante la evolución de Cerro de Pasco (1901 - 2008). Fuente: elaboración propia.

Habría que agregar que a los problemas con la minería de la plata, para cuando se fue derivando hacia el cobre (cuyo precio iba incrementándose), y poder percibir el mismo beneficio que antes, era necesario producir una mayor cantidad de cobre (Contreras, 2004), lo que generó mayores problemas con el transporte. Este fue un elemento clave que impidió la explotación a gran escala del cobre. Como lo explica George Steel, cónsul británico en Cerro de Pasco: "La explotación de mineral de cobre continúa creciendo ligeramente, y depende de los reducidos medios de comunicación con el Ferrocarril Central en La Oroya y Casapalca. El número de animales disponibles es limitado, consecuentemente la producción de las minas está subordinada a los medios de transporte" (Contreras, 2004).

Por el lado del capital social (Ks), es determinante la poca unión entre los propios mineros, lo que le decrecimiento debido a que por las continuas luchas entre los actores locales por el desarrollo del ferrocarril, no se llegaba a nada productivo. Los mineros querían construir el ferrocarril para abaratar sus costos de transporte, mientras que los arrieros no querían que se construyera porque de esa manera perderían su modo de vida y las ganancias que su negocio les daba. Pero además la banca local no se arriesgaba a realizar préstamos que ellos consideraban de riesgo a largo plazo, como es el caso de la minería, algo que impidió que los mineros locales tuvieran suficiente capital para que salieran de la trampa en la que se encontraban (Contreras, 2004).

Fines de la Etapa I - Cerro de Pasco Copper Corporation $\rightarrow$ inicios del tajo abierto

Para fines de los años 40 e inicios de los 50, la explotación era subterránea, y la ley mineral había descendido a niveles en los que la explotación del 
mismo ya no era rentable. La compañía amenazó con suspender operaciones si no le daban el permiso que requerían $\mathrm{y}$, siendo la principal empleadora de la región, motivó el reclamo de la población, quienes pidieron al Estado intervenir a favor de la empresa (Mayuntupa, 1995). Esta situación indicaba que el capital natural estaba en descenso (Kn).

La CPCC, con la propiedad de la planta concentradora de Paragsha y con el inicio de las operaciones en el tajo abierto, no se limitó a explotar cobre, sino que se diversificó y empezó a producir zinc y plomo en 1963, y el crecimiento en la escala de las operaciones mineras hizo que la población de la ciudad aumentara, registrándose en 1961 un total de 21,400 habitantes (Vega-Centeno, 2007). Este incremento en la población, así como la inversión en las nuevas plantas por parte de la CPCC significó un incremento en el capital físico (Kf).

Para cuando ocurre la fase $\Omega$ de la etapa I se iniciaba el quiebre del Kp debido a que con la decisión de permitir el tajo abierto, el tajo va a ser el factor que domine la dinámica de la ciudad y las decisiones de la población ya no van a ser tan relevantes como en ese momento inicial. El $\mathrm{Kn}$ se encontraba en descenso debido a que la ley mineral había bajado mucho y ya no era rentable seguir operaciones con esa calidad de mineral bajo ese tipo de explotación. Por eso mismo que el Ke tiene un ligero bache, que poco después es compensado por el nuevo tipo de explotación. Pero es aquí en donde el $\mathrm{Kf}$ de la ciudad entra en declive permanente ya que el nuevo sistema de explotación, para sobrevivir y mantenerse, va a tener que ir destruyendo la ciudad de a pocos, y con ello toda su infraestructura e historia, alterando también toda la dinámica socio-económica y ambiental de la misma. El Ks por otro lado, todavía sigue subiendo debido a que los aspectos sociales todavía siguen estructurados, el tajo recién empieza, y fue la población quienes, junto con la autoridades, decidieron que existiese el tajo.

Inicio de la Etapa II - El tajo como atractor de la dinámica de la ciudad

Esta segunda etapa empezó con el cambio en el modo de extraer mineral en la ciudad de Cerro de Pasco. A pesar de que en este período ocurrieron importantes cambios políticos en el Perú que trastocaron al sistema de propiedad, en el que la tenencia de la empresa minera cambió manos varias veces, el atractor principal, el tajo, sigue siendo el centro de gravedad sobre el cual gira la ciudad de Cerro de Pasco. La actividad minera continúa y el impacto del tajo se está incrementando.

Por otro lado, a partir de la década del 50 la CPCC empezó a intensificar la mecanización de la producción, en parte debido a la introducción de la minería a tajo abierto, con lo cual los salarios empezaron a subir de una manera constante y a una tasa mayor que los índices del costo de vida. Un factor importante en este incremento fue la presión de los sindicatos laborales, lo que a su vez estimuló la mecanización. Este último hecho ayudó a que se incrementara la productividad de los trabajadores, lo que repercutió en el incremento salarial a los trabajadores (Long \& Roberts, 2001). La mejora en el aspecto de la especialización, el incremento de los salarios y las mejores condiciones de los trabajadores significaron que $\mathrm{Ke}$, $\mathrm{Ks}$ y $\mathrm{Kh}$ se mantengan en alza. El capital político $(\mathrm{Kp})$ tuvo un ligero incremento debido a que la población fue la que tomó la decisión de permitir que se iniciaran las operaciones a tajo abierto (Mayuntupa, 1995), aunque una vez que el tajo empezó y tomó el control de la dinámica de la ciudad, la población perdió completamente ese poder. Con el tiempo y la expansión del tajo, la descomposición física de la ciudad va a llevar a que las estructuras sociales se vayan degradando, haya menos cohesión en todo sentido, y porque además empezaron a evolucionar polos dispersos en el aspecto físico y con sus propias características en lo que alguna vez fue una ciudad integrada. Con el tajo también empezó a disminuir la calidad de vida de la población en general (Minera Interandina de Consultores, 2006; Labor, 2007a).

En esta etapa el tajo es el atractor y no la gran empresa minera sobre la que aparentemente giraría todo. En la etapa anterior no había influencia física de la minería dentro de la ciudad y no se afectaba a los procesos dentro de la misma, aunque sí la que dominaba los procesos de toda la región. Siendo la CPCC la más grande empresa de la zona central del Perú, la gran corporación transnacional es el centro de todos los procesos que ocurren en la región central del país (Long \& Roberts, 2001; Thorp \& Bertram, 1988). Pero a partir de 1956, año en que empiezan las operaciones de tajo abierto, la ciudad va a girar en torno a lo que ocurra en el mismo, pues es una directa intromisión en sus vidas. El tajo ha movido parte de la ciudad hacia San Juan Pampa, eliminando la parte antigua de Cerro de Pasco, y cada vez que el mineral parece agotarse, el tajo debe crecer y absorber alguna otra parte de la urbe. Debido a ello, no importa qué empresa minera se encuentre laborando, el hecho es que para continuar produciendo va a tener que impactar sobre la estructura física de la ciudad.

Es en este punto en el que se discrepa con la versión de los autores Dammert \& Carhuaricra (2003), quienes determinan que con la nacionalización empieza otra etapa en el ciclo de existencia de Cerro de Pasco. La aparición de CENTROMIN sólo cambia la tenencia de las propiedades de la CPCC, pero no la dinámica que existía con respecto a la ciudad y a la población en sí. Dammert \& Carhuaricra (2003), establecen lo siguiente: 
"El cambio más importante en la explotación minera del centro fue la ruptura de la modalidad de 'mina-hacienda', ocurrida por dos razones convergentes: La reforma agraria de 1969, que tras la recuperación comunal de tierras permitió separarlas de la gran empresa minera; y, la nacionalización de la Cerro de Pasco Copper Corporation formándose la empresa estatal Centromin Perú, en 1974. Estos cambios pusieron en cuestión el desastre ecológico como método de rentabilidad empresarial. Pero no se acompañó de un manejo territorial distinto, ni se modificaron los procesos mineros que deterioraban el ambiente. La empresa Centromin, pese al esfuerzo de algunos de sus profesionales y trabajadores, continuó con los métodos impuestos por la anterior empresa Cerro de Pasco Corporation."

Testimonios sobre la diferencia en la gestión de Centromin y la CPCC muestran los dos tipos de trato hacia el trabajador y cómo el haber pasado a ser nacionalizada la empresa minera, significó más una pérdida que una ganancia para el trabajador local. En el siguiente párrafo se presentan dos testimonios de ello (De Echave, 2000):

"En realidad estuve de acuerdo con la nacionalización del gobierno de Velasco. Los gringos se llevaban todo el mineral nuestro y no pagaban nada; y a los trabajadores les pagaban bien poquito. Cuando pasó a ser Centromin hemos estado bien, los sueldos mejoraron un poco, pero poco a poco nos dimos cuenta que las cosas eran iguales y hasta empeoraron, se olvidaron de mejorar las viviendas. Las viviendas son antiguas, desde antes de la nacionalización. (Hilario Meza Alejandro y Yola Justiniano)".

Esto indica que, a pesar del cambio de dueño de la empresa minera, el tajo continuó y peor aún, las cosas empeoraron para las poblaciones locales. De esta manera el impacto de Centromin en realidad no afectó la dinámica en la que ya se encontraba la ciudad.

Fin de la etapa II - Se considera que la ciudad de Cerro de Pasco se encuentra entrando a la fase $\Omega$ del ciclo adaptativo debido a que una serie de capitales existentes en la ciudad están disminuyendo, los que se explicarán a continuación:

Capital natural.

Primero, la producción del tajo Raúl Rojas (el tajo central de la ciudad) está decayendo (Figura 2). Se puede apreciar que el $\mathrm{Pb}$ y el $\mathrm{Zn}$ tuvieron un crecimiento ligero entre los años 1995 y 1999 , mientras que en términos de $\mathrm{Ag}$ este incremento fue mayor. A partir de ese momento la producción disminuyó de manera notable, lo que quiere decir que la cantidad de mineral existente en la veta actual se está terminando. Por eso existe la presión por parte de la empresa minera Volcán por expandir el tajo hacia la zona de Chaupimarca (Plan L), hecho que permitiría mantener operativa las operaciones mineras en el lugar por un tiempo más.

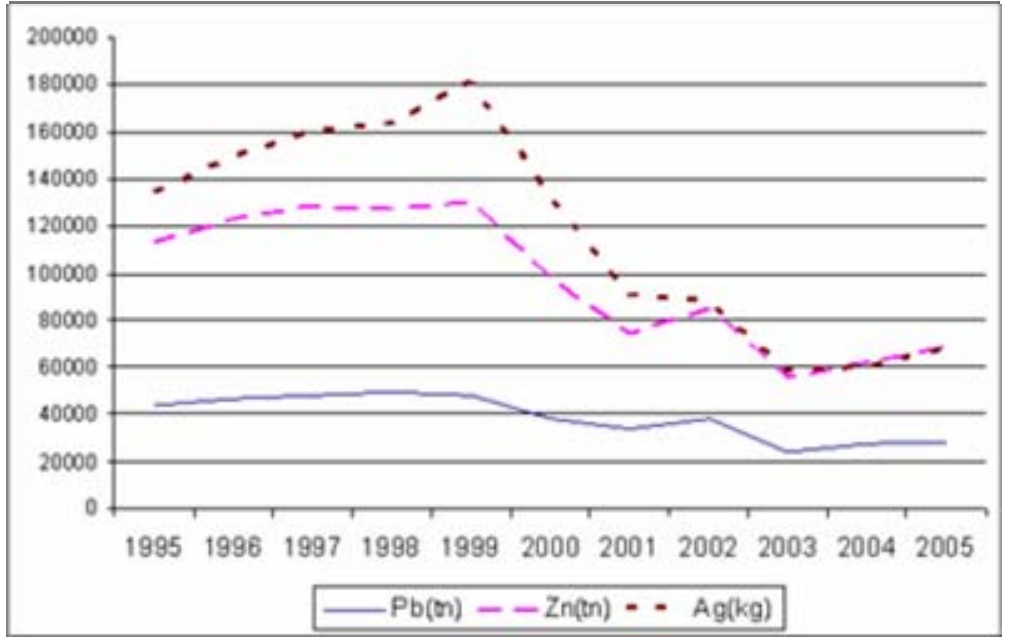

Figura 2. Producción del tajo Raúl Rojas

Fuente: Minera Interandina de Consultores SRL. (2006) elaboración propia.

Esa necesidad de expansión del tajo, mediante proyectos como el Plan L, indica que el capital natural está disminuyendo $(\mathrm{Kn} \downarrow)$. $\quad \mathrm{El} \mathrm{Kn}$ está decayendo ahora que parece que se ha llegado a un límite en la tolerancia de la población con respecto a cuánto de la ciudad están dispuestos a perder para que la minería continúe.

Capital económico.

Asimismo, la dinámica comercial local se ve afectada, con lo que el capital económico local también está disminuyendo $(\mathrm{Ke} \downarrow)$. El Ke también se está reduciendo debido a que en los últimos tiempos a consecuencia de la crisis internacional ha ocasionado la caída del precio de los metales, lo que ha reducido la posibilidad de conseguir el dinero necesario para financiar al traslado de la ciudad (Figura 3), lo que mantiene la progresiva desestructuración física de la misma.

Capital físico.

La pérdida de infraestructura el tajo ha generado desorden en el tráfico, problemas con el ordenamiento territorial urbano y pérdida de la calidad de vida en la ciudad, con lo que el capital físico se considera que ha disminuido $(\mathrm{Kf} \downarrow)$. La ciudad empezó a perder este capital desde el momento en que se empezó a trabajar el tajo, llegándose al límite en el que va a ser necesario trasladar la urbe para poder proporcionar un desarrollo adecuado a su población (Diario El Peruano, 2008).

Capital humano 


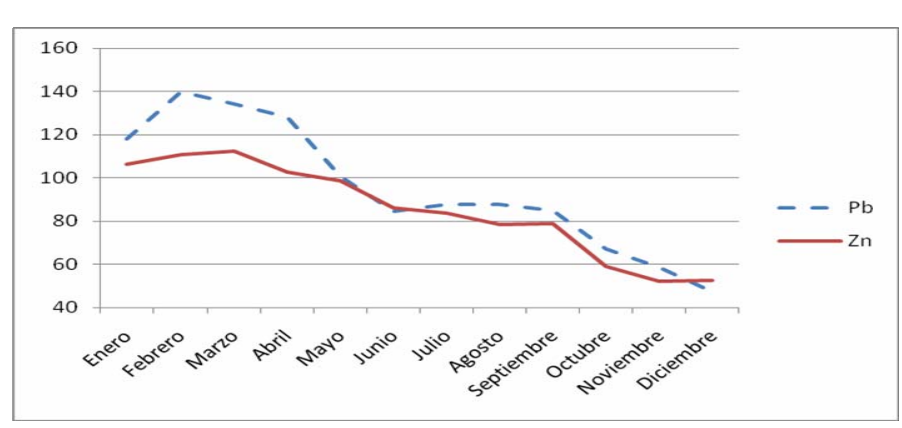

Figura 3. Caída de los precios internacionales del $\mathrm{Pb}$ y $\mathrm{Zn}$ durante el año 2008.

Elaboración propia a partir de Actualidad Minera (2009a). se puede inferir una relación muy directa entre la cercanía a las fuentes de plomo y la presencia en sangre de este metal.

Esta contaminación es debido a las fundiciones y a los procesos industriales, no al proceso del socavón, que más bien trae enfermedades a los trabajadores expuestos a las partículas o gases tóxicos que pudieran inhalar. Las fundiciones para esta fecha se encuentran fuera de la ciudad, en sitios como La Oroya. Con el inicio de las operaciones mediante el tajo abierto en 1956, la principal fuente del material particulado ahora es el centro de la ciudad y los desmontes que se hallan en los alrededores.

Otro indicador de la disminución del capital humano $(\mathrm{Kh})$ es la tendencia demográfica. Dos variables son claras indicadores de esta tendencia y de la pérdida de Kh. La primera es la cantidad de nacimientos. Esta ha disminuido de manera notable, siendo especialmente notoria en Chaupimarca y en Yanacancha, en donde el número de nacimientos bajó en 66 y en 97 respectivamente, lo que en porcentajes representa el $-7.6 \%$ y $-15.4 \%$ (Labor, 2005).

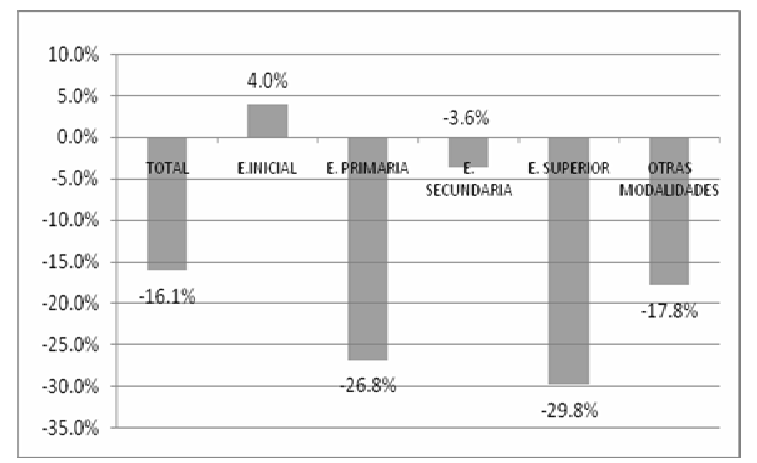

Figura 4. Alumnos matriculados en el sistema educativo escolarizado estatal y no estatal según modalidad $2002-2008$.

Fuente: INEI (2009a)

La segunda variable es el nivel de escolaridad (figura 4). En el período del 2002 al 2008 la cantidad de alumnos en el sistema educativo de la provincia de Pasco se redujo en total en un $16.1 \%$, siendo los peores casos la reducción en el número de alumnos del nivel primario $(-26.8 \%)$ y el superior $(-29.8 \%)$. Esta pérdida de elementos que en el futuro inmediato (el superior) y en el mediano plazo (primario) podrían haber aportado a la ciudad va a reducir el $\mathrm{Kh}$ de la ciudad en el corto y mediano plazo.

La disminución en la calidad de vida debido a la contaminación por metales, especialmente por plomo, la disgregación del casco urbano, la falta de servicios adecuada, y la poca posibilidad de planificar en la ciudad debido al constante cambio en su fisonomía, todas ellas reducen el Kh, así como el Ks. 
Por otro lado, la Tabla 2 muestra la evolución del índice de Necesidades Básicas Insatisfechas (NBI) (Feres \& Mancero, 2001), que arroja un incremento del 8,2\% en el período de 1993 al 2005. En un período de 12 años en los que ha habido bonanza económica y minera, un incremento en las NBI arroja un estado de empobrecimiento. Esto aunado a un bajo crecimiento poblacional y la disminución en la natalidad, es una señal de la disminución del capital humano (Kh).

Tabla 2. Necesidades básicas insatisfechas.

\begin{tabular}{lcc}
\hline \multirow{2}{*}{\multicolumn{1}{c}{ Ámbito }} & \multicolumn{2}{c}{ Hogares con NBI } \\
\cline { 2 - 3 } & 1993 & 2005 \\
\cline { 2 - 3 } Cerro de Pasco & 63,5 & 71,7 \\
Chaupimarca & 66,2 & 70,3 \\
Simón Bolívar & 87,0 & 88,3 \\
Yanacancha & 52,4 & 56,7 \\
\hline Fuente: Labor, 2007a & &
\end{tabular}

Capital social (Ks).

La expansión del tajo trae consigo la desestructuración de la zona urbana. Vittor (2007) comenta lo siguiente: El Plan L "en la práctica implica la destrucción de viviendas y bienes de uso común, así como romper con la dinámica del comercio local y con ella la memoria histórica y cultural del Cerro de Pasco", lo que quiere decir que el capital social también estaría siendo afectado de manera negativa $(\mathrm{Ks} \downarrow)$.

Se hace más difícil que las personas se puedan comunicar entre sí, del mismo modo en que lo hacían anteriormente, y por el otro, se crean divisiones entre los pobladores debido a que algunos se pueden ver beneficiados por la existencia del tajo, mientras que otros se ven tremendamente perjudicados. Pero además el cambio en la estructura laboral, con trabajadores foráneos, lleva a cambios sociales y culturales locales.

Entre otras variables que tienen influencia sobre Ks es la variable migración, que también nos indica el nivel de cohesión que Cerro de Pasco tiene. La Figura 5 nos indica el cambio en el número de migrantes entre los residentes de la ciudad. Entre 1993 y el 2007, aunque la población de la ciudad se ha incrementado, el número de residentes que son inmigrantes ha disminuido. Esto significa que la ciudad no es tan atractiva para los foráneos. Por otro lado, en la Figura 6 se puede apreciar que en Pasco es mucho mayor la cantidad de emigrantes que la de inmigrantes, aunque la brecha entre ambos se haya reducido.

En términos laborales, en

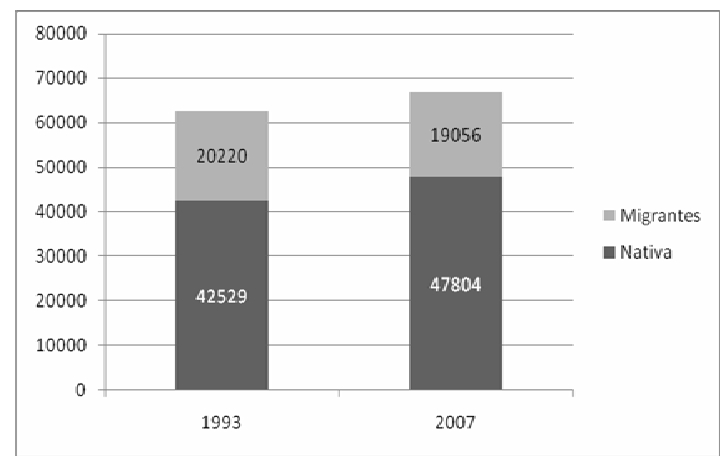

Figura 5. Número de migrantes de la población de Cerro de Pasco.

Fuente: INEI $\left(2009^{a}\right)$.

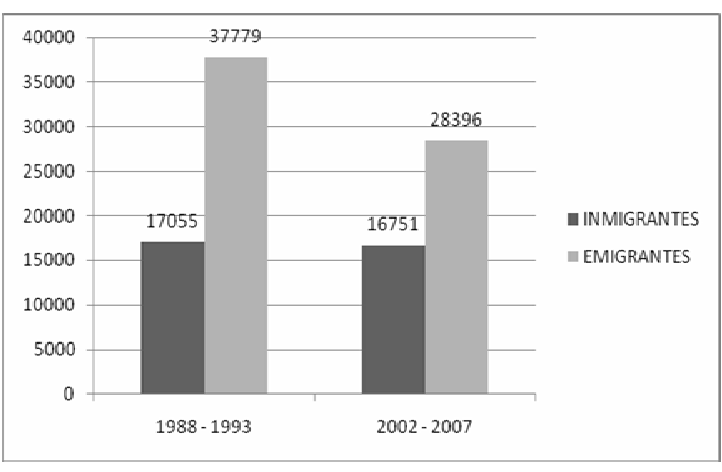

Figura 6. Inmigrantes y emigrantes en Pasco.

Fuente: INEI (2009a).

Cerro de Pasco, en el periodo entre 1990 y el 2000 fueron despedidos 1800 trabajadores, lo que considerando que cada trabajador tenga una familia de 6 , estaríamos hablando de 10,800 personas afectadas (Osorio, 2003), generándose un incremento en el nivel de pobreza e inestabilidad social y económica en la ciudad y en la región.

Por otro lado, también ha sido importante el tipo de contrato que tiene el trabajador. Además de haber una reducción en la cantidad total de trabajadores, el tipo de contrato también había cambiado de manera notable. Mientras que en el año 1992 este número se

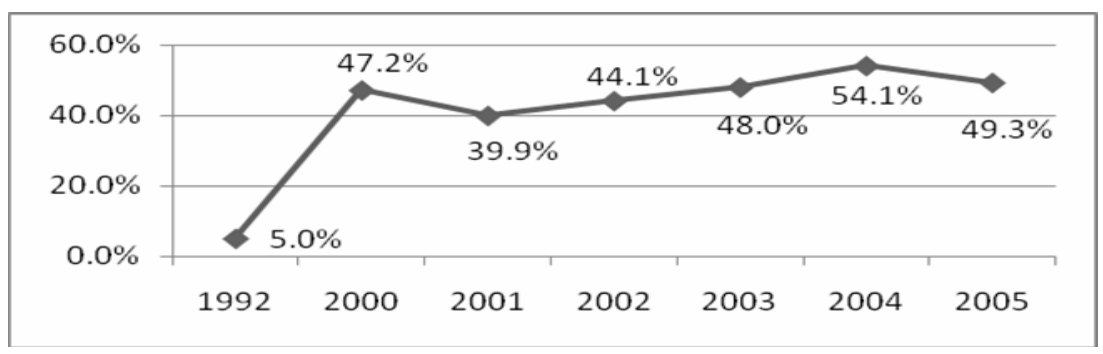

Figura 7. Porcentaje de trabajadores mineros pertenecientes a contratas. Elaboración propia. Fuente de datos: Labor (2006), Labor (2007a). 
reducía a apenas 5\%.Para el año 2002, casi el 45\% de los trabajadores mineros estaba en contratas (Figura 7). Debido a la tendencia del mercado laboral a tercerizarse, la proporción de trabajadores de contrata se incrementó a $49.3 \%$, lo que todavía es menor a lo que está ocurriendo en el mercado laboral minero. Según Consiglieri (2007) en el Perú existen aproximadamente 120,000 trabajadores mineros ( $\sin$ incluir a los informales), de los cuales 85,000 trabajan para services, contratas y subcontratas, lo que representa al 70.8\% del total del empleo formal.

El problema que esto trae al trabajador es que debilita los mecanismos directos de relación y negociación entre empresas y trabajadores mineros (Consiglieri, 2007), existe una alta precariedad de la fuerza laboral minera al mantenerse salarios reales estancados muy por debajo de los estándares internacionales. Al ser estos trabajadores de las contratas gente que generalmente no pertenece a la localidad y están de tránsito, se genera además una menor absorción de mano de obra local (Labor, 2006).
Análisis comparativo de los diferentes capitales en el tiempo

La Figura 8 muestra de manera gráfica la comparación entre los procesos más importantes ocurridos en el periodo de estudio en Cerro de Pasco, y las fluctuaciones que han ocurrido en los diferentes capitales en ese tiempo. Se ha realizado el análisis de los capitales durante el periodo de estudio para Cerro de Pasco, cómo han ido evolucionando, y cómo eso encaja con las fases $\Omega$ antes definidas como tales. Las curvas se han hecho de manera cualitativa. Las flechas nos indican los momentos en el que un capital empieza el descenso, lo que se podría considerar un punto de quiebre de ese sistema. Se señala los principales eventos que ocurrieron durante el periodo de estudio, en los que se han representado el tipo de empresa minera que existió, los principales minerales explotados y los tipos de explotación que existentes en Cerro de Pasco. Junto a ellos se hallan las fluctuaciones de los distintos capitales.

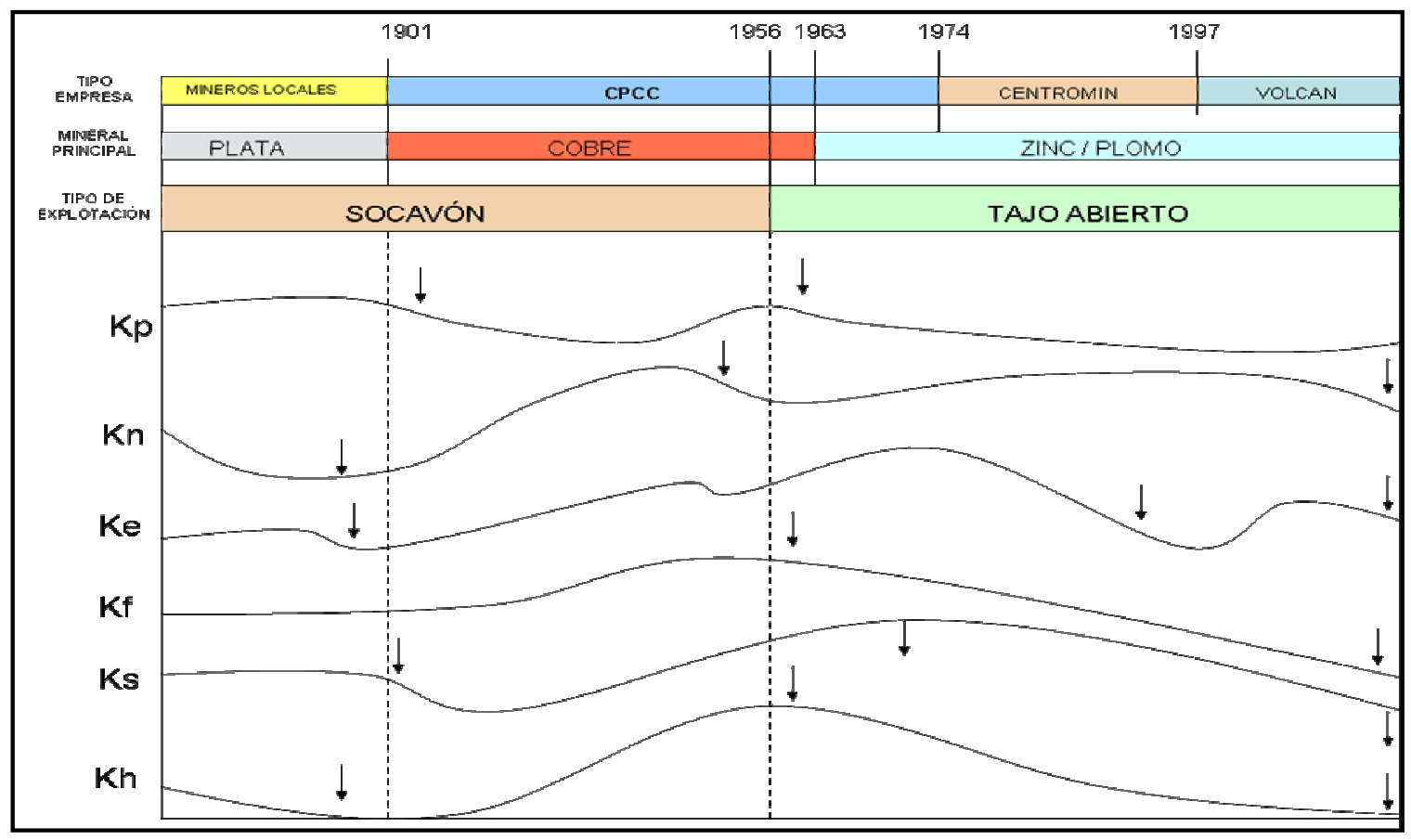

Figura 8. Fluctuación de los diferentes capitales durante el periodo de estudio.

Fuente: elaboración propia.

Capital político (Kp).

Mientras que el poder político de los sindicatos se ha visto debilitado por la menor cantidad de trabajadores en planilla y más en contratas, el Kp del sistema se ha incrementado debido a que la población de Cerro de Pasco se está oponiendo más abiertamente a la continua expansión del tajo, como es el caso de la lucha contra la aplicación del Plan L (Actualidad Minera, 2009b). Esto ha ocasionado que el Kp de la ciudad se eleve y se mantenga estancada la expansión del tajo.
De los tres momentos clave que ha habido en este periodo - 1901, 1956 y el del 2008 - el de 1956 es el que tuvo un menor número de capitales en descenso al momento de iniciarse el tajo (Kn), comparado con los otros casos que ya tenían a la mayoría de sus capitales en descenso al iniciarse la siguiente etapa, aunque la pérdida del capital físico (Kf) que ocasionó el tajo, llevó a la descomposición de varios otros capitales. La crisis de 1901 es una crisis que debido a que hubo una conjunción de pequeñas crisis en varios de los capitales, generó una crisis de todo el sistema. 
Actualmente existe crisis en varios de los capitales, lo que significa que se está dando las condiciones para que se presente una gran crisis nuevamente.

\section{Conclusiones.}

Se ha determinado un ciclo adaptativo completo y uno parcial en el conflicto socio-ambiental de Cerro de Pasco, finalizando el primero de ellos con el inicio del tajo abierto y estando el segundo entrando en una fase omega. Mediante el análisis de capitales se ha determinado además que la fase $\Omega$ de fines de la etapa I fue más leve que las fases $\Omega$ de la etapa 0 o la que está ocurriendo en la etapa II.

Se ha determinado que el análisis mediante el uso de los capitales puede permitir la determinación de los ciclos de manera más concluyente y que puede ser utilizado como herramienta para futuros trabajos que involucren sistema socio-ecológicos.

\section{Literatura citada.}

Abel T. 1998. Complex Adaptive Systems, Evolutionism, and Ecology within Anthropology: Interdisciplinary Research for Understanding Cultural and Ecological Dynamics. Journal of Ecological Anthropology 2: 6-29.

Abel N., Cumming D. \& Anderies J. 2006. Collapse and Reorganization in Social-Ecological Systems: Questions, Some Ideas, and Policy Implications. Ecology and Society 11(1):17

Actualidad Minera. 2009a. Cooperacción. Nro. 117.

Actualidad Minera. 2009b. Campaña por la defensa de la laguna de Patarcocha. Cooperacción. Nro. 118.

Barrios C. 2008. La minería en la sierra central del Perú. El desarrollo empresarial desde la perspectiva local. Red académica Iberoamericana.

Baumann P. \& Sinha S. 2001. Linking development with democratic processes in India: Political capital and sustainable livelihoods analysis. Natural Resource Perspectives - Number 68 .

Bush S. 2004. A Political Ecology of Living Aquatic Resources in LAO PDR. PhD. Thesis. School of Geosciences -University of Sydney.

Consiglieri J. 2007. La situación laboral en el sector minero. Actualidad Minera del Perú. Cooperación.

Contreras C. 2004. El aprendizaje del capitalismo - Estudios de historia económica y social del Perú republicano. Instituto de Estudios Peruanos.

Dammert M. \& Carhuaricra E. 2003. Minería y crisis socio ambiental en la región central altoandina - Pasco, Junin y Yauli - La Oroya. Cuadernos Labor 4. Asociación Civil Centro de Cultura Popular Labor.

De Echave J. 2000. Minería y comunidades - Testimonios orales y gráficos. The Panos Institute London y Cooperación.

Diario El Peruano. 13 de diciembre de 2008. Ley 29293.

Guadalupe E., Romero A., Cabrera C., De la Cruz E., Tumialán P., Campoblanco H., Blas W., Cantorin M., Campian M. \& Cevallos C. 2006. El patrimonio minero metalúrgico de Tinyahuarco. Revista del Instituto de Investigaciones FIGMMG UNMSM. 9(18): 23-31.

Feres J. \& Mancero X. 2001. El método de las necesidades básicas insatisfechas (NBI) y sus aplicaciones en
América Latina. CEPAL - ECLAC. División de estadísticas y proyecciones económicas.

Holling C.S. 2001. Understanding the Complexity of Economic, Ecological, and Social Systems. Ecosystems 4:390-405.

Instituto Nacional de Estadística e Informática (INEI). 2009a. Perú: Migraciones Internas 1993-2007.

Instituto Nacional de Estadística e Informática (INEI). 2009b. Departamento Pasco: Compendio Estadístico 2009 - Sistema regional de estadística.

Kent M. L. 1995. Los bolivianos en el tiempo. www.kalipedia.com/graficos/precio-internacional-plata1830. Acceso en enero 2009.

Labor. 2005. El aire y la salud en emergencia. Boletín participación 1. Asociación Civil Centro de Cultura Popular Labor.

Labor. 2006. Nacionalización minera y crisis del modelo estatal. Participación - Boletín Especial 2. Asociación Civil Centro de Cultura Popular Labor.

Labor. 2007 a. Boletín Participación 11. Asociación Civil Centro de Cultura Popular Labor.

Labor. 2007 b. Volcán Compañía Minera S.A.A. Participación - Boletín Especial 3. Asociación Civil Centro de Cultura Popular Labor.

Long N. \& Roberts B. 2001. Mineros, campesinos y empresarios en la sierra central del Perú. Instituto de Estudios Peruanos.

Mayuntupa E. 1995. Pasco: Bodas de Oro. Claros y Asociados S.C.R.L.

Minera Interandina de Consultores SRL. 2006. Estudio de Impacto Ambiental - Ampliación Paragsha - San Expedito. Capítulo I.

Nation J. \& Gleaves D. 2001.Low-level lead exposure and intelligence in children. Archives of Clinical Neuropsychology 16 (2001): 375-388.

Osorio V. 2003. Cerro de Pasco y la Provincia de Pasco en emergencia Ambiental. Seminario internacional "Procesos de concertación y desarrollo local en zonas mineras"

Quispe A. 2006. Balance de la Minería en Pasco. Participación - Boletín Especial 2. Labor - Pasco.

Reis J. 2001. Human capital and industrialization: The case of a late comer (Portugal 1890). European University Institute and Instituto de Ciências Sociais, Lisbon.

Resilience Alliance. 2008. http://www.resalliance.org/570.php - Acceso en noviembre 2008.

Samamé M. 1980. El Perú Minero - Tomo I. INCITEMI

Seixas C. 2002. Social-ecological dynamics in management systems: investigating a coastal lagoon fishery in southern Brazil. PhD Dissertation. Natural resources Institute. University of Manitoba.

Suárez N. 2008. Reproducción de las sociedades rurales. Revista Luna Azul No.26.

Thorp R. \& Bertram G. 1988. Perú: 1890 - 1977 Crecimiento y políticas en una economía abierta. Mosca Editores S.R.L.

Vega-Centeno P. 2007. El ocaso de un modelo de ciudad minera: Una mirada a Cerro de Pasco y La Oroya. Cuadernos 6. Departamento de Arquitectura - Pontificia Universidad Católica del Perú.

Virginia Department of Health. 2004. Lead-Elevated Blood Lead Levels in Children. 
Vittor L. 2007. Cerro de Pasco y la expansión minera, un conflicto infinito. Agencia Latinoamericana de Información. América Latina en Movimiento. www.alainet.org.
Weeks B., Rodriguez M.A. \& Blakeslee J.H. 2004. Panarchy: Complexity and Regime Change in Human Societies. Santa Fe Institute Complex Systems Summer School Proceedings.

\footnotetext{
${ }^{1}$ Ingeniero ambiental - Docente UNALM - Departamento de Ingeniería Ambiental Física y Meteorología, ajaramayob@yahoo.com

2 Profesor Principal UNALM - Departamento de Biología, esi@lamolina.edu.pe
} 